\title{
Predictors of In-hospital Outcomes after Primary Percutaneous Coronary Intervention for Acute Myocardial Infarction in Patients with a High Killip Class
}

\author{
Jun Shiraishi ${ }^{1}$, Yoshio Kohno ${ }^{1}$, Takeshi Nakamura ${ }^{2}$, Takashi Yanagiuchi ${ }^{1}$, Sho Hashimoto ${ }^{1}$, \\ Daisuke Ito ${ }^{1}$, Masayoshi Kimura ${ }^{1}$, Akihiro Matsui ${ }^{1}$, Hirokazu Yokoi ${ }^{1}$, Masayasu Arihara ${ }^{3}$, \\ Masayuki Hyogo ${ }^{1}$, Takatomo Shima ${ }^{1}$, Takahisa Sawada ${ }^{1}$, Satoaki Matoba ${ }^{2}$, \\ Hiroyuki Yamada ${ }^{2}$, Akiyoshi Matsumuro ${ }^{2}$, Takeshi Shirayama ${ }^{2}$, Makoto Kitamura ${ }^{4}$ \\ and Keizo Furukawa ${ }^{5}$; on Behalf of the AMI-Kyoto Multi-Center Risk Study Group
}

\begin{abstract}
Objective The predictors of in-hospital outcomes after primary percutaneous coronary intervention (PCI) for acute myocardial infarction (AMI) complicated with heart failure or cardiogenic shock at presentation remain unclear.

Methods Using the AMI-Kyoto Multi-Center Risk Study database, the clinical background characteristics, angiographic findings, primary PCI results, and in-hospital prognoses were retrospectively compared between primary PCI-treated AMI patients with a Killip class status of $\geq 2$ (Killip 2-4 patients, $n=390$ ) and those with a Killip class 1 status (Killip 1 patients, $n=1,057$ ).

Results The Killip 2-4 patients were more likely to have a higher age and proportion of women and exhibited a higher prevalence of previous myocardial infarction, diabetes mellitus and chronic kidney disease or anemia on admission, lower systolic blood pressure (SBP) values on admission, a higher rate of multivessels or left main trunk as the culprit artery, a larger number of diseased vessels, a lower Thrombolysis In Myocardial Infarction (TIMI) grade in the infarct-related artery (IRA) before/after primary PCI and a significantly higher in-hospital mortality rate than the Killip 1 patients. According to a multivariate analysis, age was found to be an independent positive predictor of in-hospital mortality, while admission SBP was an independent positive predictor of in-hospital survival in both groups. In contrast, anemia on admission was found to be an independent predictor of in-hospital death, while the TIMI 3 flow in the IRA after PCI was found to be an independent factor for survival in the Killip 2-4 patients, but not the Killip 1 patients.

Conclusion Anemia on admission and the final TIMI 3 flow in the IRA are critical determinants of inhospital death in AMI patients with a Killip class status of $\geq 2$ undergoing primary PCI.
\end{abstract}

Key words: primary percutaneous coronary intervention, Killip classification, anemia

(Intern Med 53: 933-939, 2014)

(DOI: 10.2169/internalmedicine.53.1144)

\section{Introduction}

Primary percutaneous coronary intervention (PCI) has been established to be a first-line therapeutic strategy for treating patients with acute myocardial infarction (AMI), and accumulating evidence has shown that primary PCI can improve the prognosis of AMI patients suffering from car-

\footnotetext{
${ }^{1}$ Department of Cardiology, Kyoto First Red Cross Hospital, Japan, ${ }^{2}$ Department of Cardiovascular Medicine, Kyoto Prefectural University School of Medicine, Japan, ${ }^{3}$ Department of Emergency Medicine, Kyoto First Red Cross Hospital, Japan, ${ }^{4}$ Department of Cardiology, Kyoto Second Red Cross Hospital, Japan and ${ }^{5}$ Department of Cardiology, Tanabe Central Hospital, Japan

Received for publication June 8, 2013; Accepted for publication December 3, 2013

Correspondence to Dr. Jun Shiraishi, risa11221998@yahoo.co.jp
} 
diogenic shock or heart failure on presentation (1-5). Although AMI patients complicated with cardiogenic shock or heart failure on hospital admission continue to have a higher risk of in-hospital death, even in the primary PCI era, in which mechanical assist devices, such as intra-aortic balloon pumping (IABP) and percutaneous cardiopulmonary support (PCPS), are readily available, the detailed clinical manifestations and predictors of the in-hospital prognosis of such patients undergoing primary PCI remain to be elucidated (1-6). The AMI-Kyoto Multi-Center Risk Study, a large multicenter observational study in which collaborating hospitals in Kyoto Prefecture collected demographic, procedural and outcome data on AMI patients, was established in 2000 to analyze the data and establish an emergency-hospital network for heart diseases in Kyoto (7). The purpose of the present study was therefore to compare the clinical background characteristics, in-hospital prognosis and determinants of inhospital outcomes in primary PCI-treated AMI patients with a Killip status of 2-4 with those observed in primary PCItreated AMI patients with a Killip status of 1 using data from the AMI-Kyoto Multi-Center Risk Study.

\section{Materials and Methods}

\section{Patient population}

From July 2006 to December 2010, 1,636 consecutive patients with a diagnosis of AMI admitted to AMI-Kyoto Multi-Center Risk Study Group Hospitals within 24 hours after the onset of AMI were enrolled in the present study. Of these patients, a total of 1,481 subjects underwent primary PCI, for whom data regarding clinical background characteristics were available in 1,447 individuals. We retrospectively compared the clinical background characteristics, coronary risk factors, angiographic findings, acute primary PCI results and in-hospital prognosis between primary PCItreated patients with a Killip status of 2-4 (Killip 2-4 patients, $n=390$ ) and those with a Killip status of 1 (Killip 1 patients, $n=1,057)$. The Killip classification was determined by the first cardiology specialist to examine the patient (8). The diagnosis of AMI required the presence of two of the following three criteria: (1) a characteristic clinical history, (2) serial changes on ECG suggesting infarction (Q-waves) or injury/ischemia (ST-segment elevation and/or depression) and (3) a transient increase in the creatine phosphokinase (CK) level to more than 2-fold the normal laboratory value (9).

\section{Data collection}

The patients' demographic information, cardiovascular history and risk factors (i.e., smoking, hypercholesterolemia, hypertension and diabetes mellitus) were recorded. Hypercholesterolemia was defined as a total cholesterol level of $\geq 220 \mathrm{mg} / \mathrm{dL}$ or the use of cholesterol-lowering agents, hypertension was defined as a systemic blood pressure of $\geq$ $140 / 90 \mathrm{mmHg}$ or the use of antihypertensive treatment and diabetes mellitus was defined as a fasting blood sugar level of $\geq 126 \mathrm{mg} / \mathrm{dL}$ or the use of specific treatment. Based on the WHO criteria, anemia was defined as an admission hemoglobin concentration of $<13.0 \mathrm{~g} / \mathrm{dL}$ for men and $<12.0 \mathrm{~g} /$ $\mathrm{dL}$ for women. Chronic kidney disease (CKD) was defined as an estimated glomerular filtration rate (eGFR) less than $60 \mathrm{~mL} / \mathrm{min} / 1.73 \mathrm{~m}^{2}$, and the eGFR was calculated based on an admission laboratory analysis using the Japanese equation: eGFR $\left(\mathrm{mL} / \mathrm{min} / 1.73 \mathrm{~m}^{2}\right)=194 \times$ Serum creatinine ${ }^{(-1.094)} \times$ Age $^{(-0.287)} \times 0.739$ (if female) (10). The systolic blood pressure (SBP)/diastolic blood pressure (DBP) on admission was defined as the first SBP/DBP recorded in the supine or sitting position just after presentation to the emergency room or outpatient clinic. All in-hospital data were transmitted to the center located at the Department of Cardiovascular Medicine in Kyoto Prefectural University School of Medicine for the analysis. The study protocol was approved by each hospital's ethics committee.

\section{Emergency coronary angiography (CAG) and reper- fusion therapy}

Emergency CAG was performed using the standard technique. The coronary flow in the infarct-related artery (IRA) was graded according to the classification used in the Thrombolysis In Myocardial Infarction (TIMI) trial. Coronary stenosis was evaluated according to the American Heart Association classification (11), and significant coronary artery stenosis was defined as a reduction of at least $75 \%$ in the internal diameter of the right, left anterior descending or left circumflex coronary arteries and their major branches or $50 \%$ in the internal diameter of the left main trunk (LMT). Non-significant stenosis was defined as coronary arterial narrowing less than that of significant stenosis. Multivessels as the culprit artery was defined as simultaneous thrombosis of multiple coronary arteries or an undetermined culprit artery in the presence of multiple diseased vessels on the initial CAG. After the culprit lesions were ascertained on CAG, primary PCI was performed according to standard techniques, with the final decision regarding the appropriate strategy in each patient left to the judgment of the physician in charge. Primary PCI was defined as a coronary intervention as the initial mode of revascularization therapy performed at the time of presentation for ST-elevation myocardial infarction (MI) or non-ST-elevation MI, including posterior MI, multi-vessel MI and LMT-culprit MI, without prethrombolytic therapy (12).

\section{End point measurements}

In-hospital death was defined as all-cause death during the admission period.

\section{Statistics}

The data are expressed as the mean \pm SD for continuous variables and number (percentage) for categorical variables. The Killip 2-4 patients and Killip 1 patients were compared using the chi-square test for discrete variables and unpaired 
Table 1. Clinical Characteristics of the Study Patients (Killip 2-4 Patients vs. Killip 1 Patients)

\begin{tabular}{lccc}
\hline & $\begin{array}{c}\text { Killip 2-4 } \\
(\mathrm{n}=390)\end{array}$ & $\begin{array}{c}\text { Killip 1 } \\
(\mathrm{n}=1057)\end{array}$ & p value \\
\hline Age (mean years \pm SD) & $70.5 \pm 13.0$ & $67.1 \pm 12.3$ & $<0.001$ \\
Male (\%) & $268(68.7)$ & $795(75.2)$ & 0.013 \\
Previous MI (\%) & $58(14.9)$ & $108(10.2)$ & 0.014 \\
Risk factors & & & \\
Smoking (\%) & $141(36.2)$ & $463(43.8)$ & 0.009 \\
Hypercholesterolemia (\%) & $154(39.5)$ & $467(44.2)$ & 0.109 \\
Hypertension (\%) & $230(59.0)$ & $621(58.8)$ & 0.939 \\
Diabetes mellitus (\%) & $145(37.2)$ & $285(27.0)$ & $<0.001$ \\
CKD (\%) & $197(50.5)$ & $324(30.7)$ & $<0.001$ \\
Anemia (\%) & $142(36.4)$ & $231(21.9)$ & $<0.001$ \\
SBP at admission (mmHg) & $114 \pm 48$ & $135 \pm 28$ & $<0.001$ \\
DBP at admission (mmHg) & $68 \pm 29$ & $80 \pm 20$ & $<0.001$ \\
\hline
\end{tabular}

MI indicates myocardial infarction; CKD: chronic kidney disease, SBP: systolic blood pressure, DBP: diastolic blood pressure

Student's $t$-test for continuous variables according to standard statistical methods. The odds ratio (OR) and 95\% confidence intervals (CI) assessing the risk of in-hospital death were estimated using univariate and multivariate analyses with a logistic regression model. Potent variables with a $\mathrm{p}$ value of $<0.05$ in the univariate analyses were entered into a multivariate analysis. In the logistic regression analysis, the TIMI flow grade was categorized into two groups: grade 3 and grade $\leq 2$ or unknown. In all analyses, statistical significance was accepted at a $\mathrm{p}$ value of $<0.05$.

\section{Results}

\section{Patient characteristics and risk factors}

The clinical characteristics and risk factors of the two groups are summarized in Table 1. The Killip 2-4 patients had a higher age and proportion of women and exhibited a higher prevalence of previous MI, diabetes mellitus and CKD or anemia on admission, lower SBP values on admission, lower DBP values on admission and a reduced prevalence of current smoking compared with the Killip 1 patients. The eGFR values $\left(59.1 \pm 24.2 \mathrm{~mL} / \mathrm{min} / 1.73 \mathrm{~m}^{2}\right.$ for the Killip 2-4 patients, $71.6 \pm 30.4$ for the Killip 1 patients) and hemoglobin levels $(13.2 \pm 2.5 \mathrm{~g} / \mathrm{dL}$ for the Killip $2-4$ patients, $13.9 \pm 2.0$ for the Killip 1 patients) on admission significantly varied between the two groups.

\section{Angiographic data}

Table 2 shows the emergency CAG data for the two groups. The Killip 2-4 patients were more likely to have LMT or multivessels and less likely to have the right coronary artery as the culprit artery than the Killip 1 patients. Approximately one-fourth of the Killip 2-4 patients had three-vessel coronary artery disease or a diseased LMT on the initial CAG.
Table 2. Angiographic Findings of the Study Patients

\begin{tabular}{lccc}
\hline & $\begin{array}{c}\text { Killip 2-4 } \\
(\mathrm{n}=390)\end{array}$ & $\begin{array}{c}\text { Killip 1 } \\
(\mathrm{n}=1057)\end{array}$ & p value \\
\hline Culprit artery & & & \\
RCA (\%) & $112(28.7)$ & $407(38.5)$ & $<0.001$ \\
LAD (\%) & $176(45.1)$ & $469(44.4)$ & \\
LCx (\%) & $43(11.0)$ & $149(14.1)$ & \\
LMT (\%) & $32(8.2)$ & $9(0.9)$ & \\
Multivessels (\%) & $27(6.9)$ & $21(2.0)$ & \\
SVG (\%) & $0(0.0)$ & $2(0.2)$ & \\
No. of diseased vessels & & & \\
1 (\%) & $166(42.6)$ & $675(63.9)$ & $<0.001$ \\
$2(\%)$ & $115(29.5)$ & $282(26.7)$ & \\
$3(\%)$ & $77(19.7)$ & $91(8.6)$ & \\
LMT (\%) & $32(8.2)$ & $9(0.9)$ &
\end{tabular}

RCA indicates the right coronary artery; LAD: left anterior descending coronary artery, LCx: left circumflex coronary artery, LMT: left main trunk, SVG: saphenous vein graft

\section{Results of coronary intervention}

Table 3 shows the results of primary PCI in the two groups. Data for the TIMI grade were available in 387 of the 390 Killip 2-4 patients and 1,044 of the 1,057 Killip 1 patients. The Killip 2-4 patients tended to have lower TIMI flow grade values before/after primary PCI than the Killip 1 patients. The Killip 2-4 patients were treated more often with IABP, PCPS and temporary pacing during the procedures and/or admission period compared with the Killip 1 patients.

\section{In-hospital outcomes}

Table 4 shows the in-hospital prognoses in the two groups. The Killip 2-4 patients had higher peak CK concentrations and a significantly higher in-hospital overall mortality rate than the Killip 1 patients. The Killip 2-4 patients also had a higher prevalence of cardiac-related death as well as death ascribed to cardiogenic shock, heart failure or ventricular fibrillation than the Killip 1 patients. In contrast, the frequency of death due to ventricular rupture did not differ significantly between the two groups. In order to assess the contribution of the clinical background characteristics, risk factors, angiographic findings and primary PCI results, univariate and multivariate logistic regression analyses were performed for overall death during hospitalization in the Killip 2-4 patients (Table 5) and Killip 1 patients (Table 6). Potent variables included age, gender, medical history (previous MI, smoking, hypercholesterolemia, hypertension and diabetes mellitus), angiographic findings (multi-vessels or LMT as the culprit artery, and a number of diseased vessels $\geq 2$ or a diseased LMT), PCI results (a TIMI 3 flow before/ immediately after primary PCI and stent placement), admission SBP and the presence of CKD or anemia on admission as categorical variables or admission hemoglobin as a con- 
Table 3. Results of Coronary Intervention in the Study Patients

\begin{tabular}{cccc}
\hline & $\begin{array}{c}\text { Killip 2-4 } \\
(\mathrm{n}=390)\end{array}$ & $\begin{array}{c}\text { Killip 1 } \\
(\mathrm{n}=1057)\end{array}$ & $\mathrm{p}$ value \\
\hline Pre TIMI grade & & & \\
0 & $243(62.8)$ & $591(56.6)$ & 0.005 \\
1 & $53(13.7)$ & $109(10.4)$ & \\
2 & $46(11.9)$ & $172(16.5)$ & \\
3 & $45(11.6)$ & $172(16.5)$ & \\
Post TIMI grade & & & \\
0 & $5(1.3)$ & $14(1.3)$ & $<0.001$ \\
1 & $6(1.6)$ & $9(0.9)$ & \\
2 & $36(9.3)$ & $42(4.0)$ & \\
3 & $340(87.9)$ & $979(93.8)$ & \\
Stent (\%) & $333(85.4)$ & $932(88.2)$ & 0.156 \\
IABP (\%) & $142(36.4)$ & $65(6.1)$ & $<0.001$ \\
PCPS (\%) & $35(9.0)$ & $1(0.1)$ & $<0.001$ \\
Pacing (\%) & $61(15.6)$ & $85(8.0)$ & $<0.001$ \\
\hline
\end{tabular}

TIMI indicates thrombolysis in myocardial infarction; IABP: intra-aortic balloon pumping, PCPS: percutaneous cardiopulmonary support

Data for the TIMI grade were available in 387 of the 390 Killip 2-4 patients and in 1,044 of the 1,057 Killip 1 patients.

tinuous variable. In both groups, age was found to be an independent predictor of in-hospital mortality, while admission SBP was found to be an independent factor for in-hospital survival (Table 5, 6). In contrast, the presence of anemia on admission/admission hemoglobin concentration and multivessels or LMT as the culprit artery were found to be independent predictors of in-hospital mortality in the Killip 2-4 patients, but not Killip 1 patients, while the acquisition of a TIMI 3 flow immediately after primary PCI and a history of smoking were found to be independent factors for inhospital survival in the Killip 2-4 patients (Table 5, 6).

\section{Discussion}

The major findings of the present multicenter study are as follows: 1. among AMI patients undergoing primary PCI, anemia on admission was an independent predictor of inhospital mortality in patients with a Killip 2-4 status, but not patients with a Killip 1 status; 2. the achievement of a TIMI 3 flow immediately after primary PCI was an independent factor for in-hospital survival in the Killip 2-4 patients, but not Killip 1 patients; and 3. the Killip 2-4 patients were more likely to experience death ascribed to cardiogenic shock, heart failure or ventricular fibrillation, resulting in a higher in-hospital mortality rate, than the Killip 1 patients, while the frequency of death due to ventricular rupture was similar between the Killip 2-4 patients and Killip 1 patients.

This study is the first to investigate the clinical manifestations and determinants of in-hospital outcomes in primary PCI-treated AMI patients with a Killip 2-4 status. In the
Table 4. In-hospital Outcomes of the Study Patients

\begin{tabular}{|c|c|c|c|}
\hline & $\begin{array}{c}\text { Killip 2-4 } \\
(\mathrm{n}=390)\end{array}$ & $\begin{array}{l}\text { Killip 1 } \\
(n=1057)\end{array}$ & $\mathrm{p}$ value \\
\hline peak CK (IU/L) & $4,969.9 \pm 7,688.1$ & $2,407.8 \pm 2,176.4$ & $<0.001$ \\
\hline Death $(\%)$ & $92(23.6)$ & $29(2.7)$ & $<0.001$ \\
\hline cardiac-related (\%) & $65(16.7)$ & $15(1.4)$ & $<0.001$ \\
\hline shock & 46 & 5 & $<0.001$ \\
\hline heart failure & 10 & 3 & $<0.001$ \\
\hline rupture & 5 & 7 & 0.249 \\
\hline VF & 4 & 0 & $<0.001$ \\
\hline noncardiac-related $(\%)$ & $27(6.9)$ & $14(1.3)$ & $<0.001$ \\
\hline \multicolumn{4}{|c|}{ CK indicates creatine phosphokinase; VF: ventricular fibrillation } \\
\hline
\end{tabular}

present study, the Killip 2-4 patients had a higher in-hospital mortality rate and significantly higher prevalence of cardiacrelated deaths due to cardiogenic shock, congestive heart failure or ventricular fibrillation than the Killip 1 patients. In contrast, the frequency of cardiac rupture-related death did not vary between the groups. Hypertension, an advanced age, female gender, lower body mass index, delayed reperfusion, first AMI, one-vessel disease with a preserved left ventricular function, totally occluded LAD as the culprit vessel and small myocardial infarction due to branch occlusion have been reported to be risk factors for cardiac rupture (13-15). Although, in the present study, data regarding non-fatal cardiac rupture and the left ventricular function were lacking, the risk of ventricular rupture in the Killip 1 patients, who exhibited a lack of signs of heart failure and/ or shock, smaller peak CK values and a higher prevalence of one-vessel disease, was almost equal to that observed in the Killip 2-4 patients, who demonstrated a higher age and higher proportion of women. In addition, noncardiac-related death was also more frequent among the Killip 2-4 patients than among the Killip 1 patients. Although there were no data regarding the details of noncardiac death, noncardiacrelated death can involve underlying diseases, such as malignancy, inflammation and/or infection, that are frequently accompanied by anemia.

The present report demonstrated for the first time that the presence of anemia on admission is an independent predictor of in-hospital mortality in the primary PCI-treated AMI patients with a Killip 2-4 status, but not those with a Killip 1 status. Several previous reports have shown anemia on admission to be an independent risk factor for in-hospital death in AMI patients, including those undergoing primary PCI $(16,17)$. Anemia deteriorates myocardial ischemia as well as heart failure due to a decreased oxygen supply to the myocardium as well as increased myocardial oxygen demands resulting from a larger stroke volume and higher heart rate. However, the precise mechanisms by which the adverse effects of anemia on in-hospital outcomes are augmented, especially in AMI patients with a Killip 2-4 status remain uncertain. A recent report by Polish investigators 
Table 5. Predictors of In-hospital Mortality among the Killip 2-4 Patients (Univariate and Multivariate Logistic Regression Analyses)

\begin{tabular}{|c|c|c|c|c|c|c|c|c|c|}
\hline & \multicolumn{3}{|c|}{ Univariate } & \multicolumn{3}{|c|}{ Multivariate (model 1) } & \multicolumn{3}{|c|}{ Multivariate (model 2) } \\
\hline & OR & $95 \% \mathrm{CI}$ & $\mathrm{p}$ value & OR & $95 \% \mathrm{CI}$ & $\mathrm{p}$ value & OR & $95 \% \mathrm{CI}$ & $\mathrm{p}$ value \\
\hline Male & 0.494 & $0.304-0.801$ & 0.0043 & 0.831 & $0.440-1.570$ & 0.5686 & 0.979 & $0.509-1.881$ & 0.9488 \\
\hline Age & 1.045 & $1.023-1.067$ & $<0.0001$ & 1.031 & $1.002-1.060$ & 0.0339 & 1.031 & $1.002-1.060$ & 0.0347 \\
\hline Previous MI & 1.728 & $0.942-3.171$ & 0.0771 & & & & & & \\
\hline Smoking & 0.346 & 0.197-0.609 & 0.0002 & 0.435 & $0.221-0.859$ & 0.0164 & 0.451 & $0.228-0.890$ & 0.0216 \\
\hline Hyperlipidemia & 0.637 & $0.388-1.047$ & 0.0750 & & & & & & \\
\hline Hypertention & 0.780 & $0.487-1.250$ & 0.3026 & & & & & & \\
\hline Diabetes Mellitus & 1.184 & $0.733-1.911$ & 0.4906 & & & & & & \\
\hline Multi-vessels or LMT as culprit & 4.900 & $2.739-8.767$ & $<0.0001$ & 3.243 & $1.536-6.847$ & 0.0020 & 3.102 & $1.475-6.526$ & 0.0028 \\
\hline No. of diseased vessels $\geq 2$ or LMT & 2.105 & $1.272-3.481$ & 0.0037 & 1.519 & $0.791-2.916$ & 0.2092 & 1.481 & $0.772-2.838$ & 0.2371 \\
\hline TIMI 3 before PCI & 0.789 & $0.365-1.706$ & 0.5472 & & & & & & \\
\hline TIMI 3 after PCI & 0.222 & $0.120-0.412$ & $<0.0001$ & 0.161 & $0.074-0.350$ & $<0.0001$ & 0.177 & $0.082-0.380$ & $<0.0001$ \\
\hline Stent usage & 0.758 & $0.403-1.426$ & 0.3896 & & & & & & \\
\hline Admission anemia & 3.391 & $2.090-5.502$ & $<0.0001$ & 2.476 & $1.335-4.590$ & 0.0040 & & & \\
\hline Admission $\mathrm{Hb}$ & 0.755 & $0.683-0.834$ & $<0.0001$ & & & & 0.830 & $0.729-0.944$ & 0.0047 \\
\hline Admission SBP & 0.986 & $0.981-0.991$ & $<0.0001$ & 0.987 & $0.981-0.993$ & $<0.0001$ & 0.987 & $0.981-0.993$ & $<0.0001$ \\
\hline Admission CKD & 2.836 & $1.721-4.673$ & $<0.0001$ & 1.421 & $0.770-2.621$ & 0.2612 & 1.412 & $0.766-2.604$ & 0.2685 \\
\hline
\end{tabular}

Model 1. Adjusted for variables with a p value of $<0.05$ in the univariate analyses including anemia on admission as a categorical variable. Model 2. Adjusted for variables with a $\mathrm{p}$ value of $<0.05$ in the univariate analyses including the admission hemoglobin concentration as a continuous variable.

OR indicates the odds ratio; CI: confidence interval, MI: myocardial infarction, LMT: left main trunk, TIMI: thrombolysis in myocardial infarction, PCI: percutaneous coronary intervention, Hb: hemoglobin, SBP: systolic blood pressure, CKD: chronic kidney disease

Table 6. Predictors of In-hospital Mortality among the Killip 1 Patients (Univariate and Multivariate Logistic Regression Analyses)

\begin{tabular}{|c|c|c|c|c|c|c|c|c|c|}
\hline & \multicolumn{3}{|c|}{ Univariate } & \multicolumn{3}{|c|}{ Multivariate (model 1) } & \multicolumn{3}{|c|}{ Multivariate (model 2) } \\
\hline & OR & $95 \% \mathrm{CI}$ & $\mathrm{p}$ value & OR & $95 \% \mathrm{CI}$ & $\mathrm{p}$ value & OR & $95 \% \mathrm{CI}$ & $\mathrm{p}$ value \\
\hline Male & 0.455 & $0.214-0.966$ & 0.0404 & 0.889 & $0.369-2.141$ & 0.7935 & 1.027 & $0.420-2.513$ & 0.9538 \\
\hline Age & 1.073 & $1.035-1.113$ & 0.0001 & 1.049 & $1.005-1.095$ & 0.0275 & 1.050 & $1.005-1.096$ & 0.0278 \\
\hline Previous MI & 1.422 & $0.485-4.165$ & 0.5212 & & & & & & \\
\hline Smoking & 0.399 & $0.169-0.943$ & 0.0362 & 0.594 & $0.220-1.605$ & 0.3046 & 0.626 & $0.232-1.691$ & 0.3554 \\
\hline Hyperlipidemia & 0.321 & $0.130-0.795$ & 0.0140 & 0.492 & $0.190-1.274$ & 0.1438 & 0.500 & $0.193-1.301$ & 0.1554 \\
\hline Hypertention & 0.561 & $0.267-1.179$ & 0.1273 & & & & & & \\
\hline Diabetes Mellitus & 0.858 & $0.363-2.032$ & 0.7283 & & & & & & \\
\hline Multi-vessels or LMT as culprit & 4.278 & $1.220-15.004$ & 0.0232 & 4.150 & $0.985-17.482$ & 0.0524 & 4.014 & $0.956-16.848$ & 0.0575 \\
\hline No. of diseased vessels $\geq 2$ or LMT & 0.928 & $0.427-2.017$ & 0.8506 & & & & & & \\
\hline TIMI 3 before PCI & 0.374 & $0.088-1.587$ & 0.1822 & & & & & & \\
\hline TIMI 3 after PCI & 0.367 & $0.136-0.990$ & 0.0477 & 0.315 & $0.100-0.990$ & 0.0481 & 0.336 & $0.108-1.045$ & 0.0596 \\
\hline Stent usage & 0.408 & $0.170-0.975$ & 0.0436 & 0.556 & $0.210-1.470$ & 0.2368 & 0.558 & $0.211-1.475$ & 0.2396 \\
\hline Admission anemia & 4.028 & $1.915-8.473$ & 0.0002 & 2.178 & $0.969-4.894$ & 0.0596 & & & \\
\hline Admission $\mathrm{Hb}$ & 0.712 & $0.609-0.832$ & $<0.0001$ & & & & 0.864 & $0.714-1.045$ & 0.1328 \\
\hline Admission SBP & 0.970 & $0.959-0.982$ & $<0.0001$ & 0.974 & $0.961-0.986$ & $<0.0001$ & 0.975 & $0.962-0.987$ & 0.0001 \\
\hline Admission CKD & 2.877 & $1.367-6.054$ & 0.0054 & 1.089 & $0.462-2.567$ & 0.8459 & 1.129 & $0.479-2.663$ & 0.7818 \\
\hline
\end{tabular}

Model 1. Adjusted for variables with a p value of $<0.05$ in the univariate analyses including admission anemia as a categorical variable. Model 2 . Adjusted for variables with a $\mathrm{p}$ value of $<0.05$ on the univariate analyses including the admission hemoglobin concentration as a continuous variable. OR indicates the odds ratio; CI: confidence interval, MI: myocardial infarction, LMT: left main trunk, TIMI: thrombolysis in myocardial infarction, PCI: percutaneous coronary intervention, Hb: hemoglobin, SBP: systolic blood pressure, CKD: chronic kidney disease

demonstrated that, in AMI patients treated with PCI in the acute phase, the presence of anemia on admission is independently associated with higher short- and long-term mor- tality rates, particularly in subgroups with multivessel disease and incomplete revascularization (18). According to that report, among AMI patients with left ventricular dys- 
function (ejection fraction $<40 \%$ ), the presence of anemia on admission tends to be associated with long-term mortality (HR 1.61, 95\% CI 1.36-1.86, $\mathrm{p}=0.0578$ ) (18). Therefore, it is reasonable to propose that the adverse prognostic effects of anemia are enhanced, especially in Killip 2-4 patients, who exhibit a higher prevalence of multivessel disease and a TIMI flow grade of $\leq 2$ in the IRA just after PCI, resulting in hemodynamic compromise and residual myocardial ischemia.

Another potential explanation for the significant adverse prognostic effects of anemia on admission observed exclusively in Killip 2-4 patients is the presence of cardiorenal anemia syndrome. Cardiorenal anemia syndrome, in which the simultaneous occurrence of CKD, anemia and heart failure creates pathological reciprocal connections, thereby resulting in an adverse impact on morbidity and mortality, has recently received greater attention $(19,20)$. Both CKD and anemia activate multiple pathophysiological pathways, including the sympathetic nervous system, renin-angiotensinaldosterone system, oxidative stress response and inflammation, resulting in the progression of heart failure (21). In the present report, although the presence of CKD on admission was not found to be an independent risk factor for inhospital death in the Killip 2-4 patients in the multivariate analysis, the Killip 2-4 patients exhibited lower eGFR values and a higher prevalence of CKD on admission than the Killip 1 patients. The role of the concept of "cardiorenal anemia syndrome" in the pathogenesis of AMI remains unclear; however, cardiorenal anemia syndrome may contribute in part to the enhanced adverse prognostic effects of anemia, particularly in AMI patients with a Killip 2-4 status.

The present findings suggest that careful and aggressive attempts to obtain a TIMI 3 flow in the IRA should be made in AMI patients with a Killip 2-4 status. Primary stenting combined with adjunctive therapy, such as the intracoronary administration of nicorandil, verapamil or nitroprusside and the intravenous infusion of nicorandil or newer antiplatelet agents, may play an important role in achieving not only an optimal epicardial coronary blood flow but also adequate peripheral microcirculation in AMI patients with congestive heart failure or cardiogenic shock $(22,23)$. Additionally, although the effects of the routine use of aspiration catheters or distal protection devices to minimize distal embolization of thrombi and plaque debris and achieve an optimal final flow in the IRA remain controversial, these devices may be necessary, especially in high-risk AMI patients (24-27). Further studies are therefore required to examine the appropriate PCI strategy for obtaining an optimal final coronary flow in the IRA in this population.

\section{Study limitations}

First, this study is a retrospective observational analysis of a relatively small number of patients. Second, data regarding clinical background characteristics and angiographic results of primary PCI were not available for all study participants. Third, we did not have adequate detailed data regarding the left ventricular function, elapsed time, ischemic time and inhospital medical treatments, which may be a predictive risk factors for in-hospital death. Fourth, "ST-elevation MI" was not discriminated from "non-ST-elevation MI." Fifth, we did not have adequate data regarding underlying diseases in the patients with noncardiac-related death and non-fatal major adverse cardiovascular events during the admission period. Sixth, hemodialysis patients were not separated from CKD patients. Seventh, the peak CK value may not precisely reflect the amount of myocardial necrosis, as patients with cardiopulmonary arrest on arrival, those undergoing frequent electrical defibrillation and those with ischemia/necrosis in the lower extremities due to the use of IABP/PCPS were included among the present real-world unselected patients. Therefore, it may be more appropriate to exclude these patients from the study population.

\section{Conclusion}

The present study provides evidence that the presence of anemia on admission is an independent risk factor for inhospital death, while a final TIMI 3 flow in the IRA is an independent factor for in-hospital survival, among primary PCI-treated AMI patients with a Killip class status of $\geq 2$. Further detailed prospective long-term follow-up studies are needed to examine the prognostic effects of anemia on admission and a final TIMI 3 flow in Japanese AMI patients with a Killip class status of $\geq 2$.

The authors state that they have no Conflict of Interest (COI).

\section{Appendix}

The following institutions and principal investigators participated in the present study as the AMI-Kyoto Multi-Center Risk Study Group: Kyoto City Hospital: Okada T., Shima M., Kiyama M., Takamiya A., Nakajima N., Matsunaga S.; Kyoto Kidugawa Hospital: Miyanaga H., Nakagawa T., Matsui H., Kunieda Y.; Kyoto Second Red Cross Hospital: Fujita H., Inoue K., Matsuo A., Kimura S., Sakatani T., Isodono K., Tsubakimoto Y.; Social Insurance Kyoto Hospital: Yamada C., Tanabe S., Yamahara Y., Katamura M.; Tanabe Central Hospital: Kusuoka S., Nishio M., Nishizawa S.; Nantan General Hospital: Tatsumi T., Keira N., Nomura T.; Ayabe Municipal Hospital: Shiga K., Kohno Y., Adachi Y.; Maizuru Medical Center: Harada Y., Hikosaka T., Nakagami T.; Kyoto Saiseikai Hospital: Ishibashi K.; Gakkentoshi Hospital: Sakai R.; Kouseikai Takeda Hospital: Matsumoto K., Kinoshita N., Sawanishi T., Nakamura R.; Kameoka Municipal Hospital: Matsuo R.; Aiseikai Yamashina Hospital: Shirai H., Kosugi Y.; Fukuchiyama Municipal Hospital: Sakamoto T.; Yamashiro Public Hospital, Tomiyasu K.; Saiseikai Shiga Hospital: Nakamura T., Nakahara Y., Kurata H., Hadase M.; Omihachiman Community Medical Center: Maki K., Tatsukawa H., Zen K., Kambayashi D.; Kyoto Prefectural Yosanoumi Hospital: Takada H.; Kyoto Prefectural University School of Medicine: Shiraishi H., Yamano T. 


\section{References}

1. Moosvi AR, Khaja F, Villanueva L, Gheorghiade M, Douthat L, Goldstein S. Early revascularization improves survival in cardiogenic shock complicating acute myocardial infarction. J Am Coll Cardiol 19: 907-914, 1992.

2. Antoniucci D, Valenti R, Santoro GM, et al. Systematic direct angioplasty and stent-supported direct angioplasty therapy for cardiogenic shock complicating acute myocardial infarction: inhospital and long-term survival. J Am Coll Cardiol 31: 294-300, 1998.

3. Hochman JS, Sleeper LA, Webb JG, et al. Early revascularization in acute myocardial infarction complicated by cardiogenic shock. SHOCK Investigators. Should We Emergently Revascularize Occluded Coronaries for Cardiogenic Shock. N Engl J Med 341: 625-634, 1999.

4. Dauerman HL, Goldberg RJ, White K, et al; GRACE Investigators. Revascularization, stenting, and outcomes of patients with acute myocardial infarction complicated by cardiogenic shock. Am J Cardiol 90: 838-842, 2002.

5. Santoro GM, Carrabba N, Migliorini A, Parodi G, Valenti R. Acute heart failure in patients with acute myocardial infarction treated with primary percutaneous coronary intervention. Eur J Heart Fail 10: 780-785, 2008.

6. DeGeare VS, Boura JA, Grines LL, O'Neill WW, Grines CL. Predictive value of the Killip classification in patients undergoing primary percutaneous coronary intervention for acute myocardial infarction. Am J Cardiol 87: 1035-1038, 2001.

7. Shiraishi J, Kohno Y, Sawada T, et al. Prognostic impact of pulse pressure at admission on in-hospital outcome after primary percutaneous coronary intervention for acute myocardial infarction. Heart Vessels 28: 434-441, 2013.

8. Killip T 3rd, Kimball JT. Treatment of myocardial infarction in a coronary care unit. A two year experience with 250 patients. Am J Cardiol 20: 457-464, 1967.

9. Gillum RF, Fortmann SP, Prineas RJ, Kottke TE. International diagnostic criteria for acute myocardial infarction and acute stroke. Am Heart J 108: 150-158, 1984.

10. Matsuo S, Imai E, Horio $M$, et al; Collaborators developing the Japanese equation for estimated GFR. Revised equations for estimated GFR from serum creatinine in Japan. Am J Kidney Dis 53: 982-992, 2009.

11. Austen WG, Edwards JE, Frye RL, et al. A reporting system on patients evaluated for coronary artery disease. Report of the Ad Hoc Committee for Grading of Coronary Artery Disease, Council on Cardiovascular Surgery, American Heart Association. Circulation 51: 5-40, 1975.

12. The GUSTO IIb Investigators. A clinical trial comparing primary coronary angioplasty with tissue plasminogen activator for acute myocardial infarction. The Global Use of Strategies to Open Occluded Coronary Arteries in Acute Coronary Syndromes (GUSTO IIb) Angioplasty Substudy Investigators. N Engl J Med 336: 1621 1628, 1997.
13. Wehrens XH, Doevendans PA. Cardiac rupture complicating myocardial infarction. Int J Cardiol 95: 285-292, 2004.

14. Yip HK, Wu CJ, Chang HW, et al. Cardiac rupture complicating acute myocardial infarction in the direct percutaneous coronary intervention reperfusion era. CHEST 124: 565-571, 2003.

15. Ino $\mathrm{Y}$, Kubo $\mathrm{T}$, Tomobuchi $\mathrm{Y}$, et al. Branch segment occlusion with acute myocardial infarction is a risk for left ventricular free wall rupture. Circ J 73: 1473-1478, 2009.

16. Sabatine MS, Morrow DA, Giugliano RP, et al. Association of hemoglobin levels with clinical outcomes in acute coronary syndromes. Circulation 111: 2042-2049, 2005.

17. Nikolsky E, Aymong ED, Halkin A, et al. Impact of anemia in patients with acute myocardial infarction undergoing primary percutaneous coronary intervention: analysis from the Controlled Abciximab and Device Investigation to Lower Late Angioplasty Complications (CADILLAC) Trial. J Am Coll Cardiol 44: 547553, 2004.

18. Kurek T, Lenarczyk R, Kowalczyk J, et al. Effect of anemia in high-risk groups of patients with acute myocardial infarction treated with percutaneous coronary intervention. Am J Cardiol 105: 611-618, 2010.

19. Silverberg DS, Wexler D, Iaina A, Steinbruch S, Wollman Y, Schwartz D. Anemia, chronic renal disease and congestive heart failure--the cardio renal anemia syndrome: the need for cooperation between cardiologists and nephrologists. Int Urol Nephrol 38: 295-310, 2006.

20. van der Meer P, van Veldhuisen DJ. Anaemia and renal dysfunction in chronic heart failure. Heart 95: 1808-1812, 2009.

21. Bongartz LG, Cramer MJ, Doevendans PA, Joles JA, Braam B. The severe cardiorenal syndrome: 'Guyton revisited.' Eur Heart J 26: 11-17, 2005.

22. Grines CL, Cox DA, Stone GW, et al. Coronary angioplasty with or without stent implantation for acute myocardial infarction. Stent Primary Angioplasty in Myocardial Infarction Study Group. N Engl J Med 341: 1949-1956, 1999.

23. Rezkalla SH, Kloner RA. No-reflow phenomenon. Circulation 105: 656-662, 2002.

24. Kaltoft A, Bøttcher M, Nielsen SS, et al. Routine thrombectomy in percutaneous coronary intervention for acute ST-segmentelevation myocardial infarction: a randomized, controlled trial. Circulation 114: 40-47, 2006.

25. Svilaas T, Vlaar PJ, van der, Horst IC, et al. Thrombus aspiration during primary percutaneous coronary intervention. $\mathrm{N}$ Engl J Med 358: 557-567, 2008.

26. Stone GW, Webb J, Cox DA, et al. Distal microcirculatory protection during percutaneous coronary intervention in acute STsegment elevation myocardial infarction: a randomized controlled trial. JAMA 293: 1063-1072, 2005.

27. Gick M, Jander N, Bestehorn HP, et al. Randomized evaluation of the effects of filter-based distal protection on myocardial perfusion and infarct size after primary percutaneous catheter intervention in myocardial infarction with and without ST-segment elevation. Circulation 112: 1462-1469, 2005.

(C) 2014 The Japanese Society of Internal Medicine

http://www.naika.or.jp/imonline/index.html 